\title{
Prevalence, type and concentration of human enterovirus and parechovirus in cerebrospinal fluid samples of pediatric patients over a 10-year period: a retrospective study
}

Silke Vollbach ${ }^{1}$, Andreas Müller ${ }^{2}$, Jan Felix Drexler ${ }^{1}$, Arne Simon ${ }^{2,4}$, Christian Drosten ${ }^{1}$, Anna Maria Eis-Hübinger ${ }^{1}$ and Marcus Panning ${ }^{1,3^{*}}$

\begin{abstract}
Background: Human enterovirus (EV) and parechovirus ( $\mathrm{HPeV}$ ) are significant causes of encephalitis and meningitis in children. The aim of this study was to determine the prevalence, type and viral RNA concentration of EV and $\mathrm{HPeV}$ in cerebrospinal fluid (CSF) samples in an unselected cohort of patients $<18$ years admitted to Bonn university hospital from 1998 to 2008.

Methods: A total of 327 CSF samples from 327 patients were retrospectively tested by real-time reverse-transcriptase PCR (RT-PCR) for EV and HPeV, and by real-time PCR for cytomegalovirus (CMV), herpes simplex virus 1/2 (HSV), and varizella zoster-virus (VZV). Samples had been submitted for routine virological work-up due to suspected meningitis or encephalitis and had been stored at $-20^{\circ} \mathrm{C}$ hereafter. Positive samples for EV and HPeV were sequenced within the gene encoding the VP1 region (EV), the VP2 and the VP3NP1 junction region ( $\mathrm{HPeV}$.

Results: The overall prevalence was 4.3 \% (14/327) for EV, 0.6 \% (2/327) for HPeV, and $0.3 \%$ (1/327) for HSV and VZV, respectively. CMV was not detected in this cohort. In children less than 3 months of age the prevalence was $7.7 \%$ (2/26) for EV and $7.7 \%$ (2/26) for HPeV, respectively. Frequency of EV detection ranged from 0 to $12 \%$ per year and highest rates were observed from June to September. All typed EV belonged to species B. Both HPeV infections were detected in the fall of 2008 and were typed as HPeV genotype 3. Viral RNA concentrations were highest in patients with HPeV infection, followed by echovirus 30 and other EV. In total, 86 \% (12/14) of EV infections presented as aseptic meningitis, whereas both $\mathrm{HPeV}$ infections presented as severe sepsis-like illness.
\end{abstract}

Conclusions: $\mathrm{EV}$ and $\mathrm{HPeV}$ were equally prevalent in children $<3$ months of age. Beyond the detection of EV and $\mathrm{HPeV}$, the determination of viral RNA concentration and typing of EV and HPeV might prove beneficial for patient management and public health.

Keywords: Human parechovirus, Enterovirus, Cerebrospinal fluid, Prevalence, Viral RNA concentration

\footnotetext{
*Correspondence: marcus.panning@uniklinik-freiburg.de

${ }^{1}$ Institute of Virology, University of Bonn Medical Centre, Sigmund-Freud Str.

25, 53105 Bonn, Germany

${ }^{3}$ Institute of Virology, Medical Center - University of Freiburg,

Hermann-Herder Str. 11, 79104 Freiburg, Germany

Full list of author information is available at the end of the article
}

(c) 2015 Vollbach et al. Open Access This article is distributed under the terms of the Creative Commons Attribution 4.0 International License (http://creativecommons.org/licenses/by/4.0/), which permits unrestricted use, distribution, and reproduction in any medium, provided you give appropriate credit to the original author(s) and the source, provide a link to the Creative Commons license, and indicate if changes were made. The Creative Commons Public Domain Dedication waiver (http://creativecommons.org/publicdomain/zero/1.0/) applies to the data made available in this article, unless otherwise stated. 


\section{Background}

Human enteroviruses (EV) and parechovirus (HPeV) belong to the highly diversified family Picornaviridae and constitute an important cause of central nervous system (CNS) infections, meningitis and encephalitis, and sepsis-like illness in children worldwide [1-3]. EV can be grouped into 4 genetically distinct species, EV-A to D. The most commonly detected EV associated with meningitis belong to EV species $\mathrm{B}$ (including echoviruses, coxsackievirus (CV)-A9, and CV-B). Continued surveillance and monitoring of EV evolution is important not only for public health (EV disease association, identification of novel variants), but also for patient management (isolation measures, treatment with IVIG). In 1997 an EV-A71 sparked an ongoing epidemic across the Asia-Pacific region and is associated with hand, foot, and mouth disease accompanied by severe neurological complications [4]. Recently, an EVD68 emerged and caused considerable morbidity and mortality among children worldwide [5, 6]. For $\mathrm{HPeV}$ at least 16 genotypes have been described to date, most of them only recently (www.picornastudygroup.com). In particular, $\mathrm{HPeV}$ genotype 3 (HPeV3) infections tend to be more severe (including sepsis-like illness) compared to infections with other $\mathrm{HPeV}$ genotypes [7].

The mainstay of laboratory diagnostics for $\mathrm{EV}$ and $\mathrm{HPeV}$ infection depends on real-time reverse-transcription-PCR (RT-PCR), which has considerably improved the detection of EV as well as $\mathrm{HPeV}$ [8]. However, $\mathrm{HPeV}$ infection is still reported infrequently and is most likely under-diagnosed in Germany and elsewhere. Information on the epidemiology over longer time periods and virus concentrations for $\mathrm{EV}$ and especially $\mathrm{HPeV}$ in cerebrospinal fluid (CSF) samples is lacking at large.

The aim of this study was to determine the prevalence, types and RNA concentrations of $\mathrm{EV}$ and $\mathrm{HPeV}$ in an unselected panel of CSF samples collected from pediatric patients with a clinical suspicion of meningitis/encephalitis or late-onset sepsis with neurological symptoms admitted at a large university hospital over a 10 -year period to assess the role of these pathogens in pediatric disease.

\section{Results}

Median patient age was 8.0 years (range, 8 days to 17 years). Eight percent of patients (26/327) were under the age of 3 months. An overall prevalence of $5.5 \%(18 / 327)$ for any viral pathogen was determined by real-time reversetranscriptase PCR (EV, $\mathrm{HPeV}$ ) and real-time PCR (CMV, HSV, VZV), respectively. EV was the most prevalent pathogen with $3.4 \%(11 / 327)$ using the assay described by Verstrepen et al. [9]. Upon use of the assay by Dierssen et al. [10] prevalence increased by a quarter to $4.3 \%$ (14/ 327) (9 male, 5 female). In children less than 3 months of age $7.7 \%(2 / 26)$ tested positive for EV with both EV assays (Verstrepen et al. and Diessen et al.) yielding congruent positive results. For $\mathrm{HPeV}$ an overall prevalence of $0.6 \%(2 / 327)$ was determined (one female, one male). Both HPeV RT-PCR assays applied yielded congruent results. If children older than 3 months of age were excluded from the analysis, the prevalence of $\mathrm{HPeV}$ raised to $7.7 \%$ $(2 / 26)$ similar to that of EV. One individual patient was positive for HSV-1 (6 year-old male) and another for VZV (4 year-old male) as determined by real-time PCR yielding an overall prevalence of $0.3 \%$ for each virus. Clinical information was lacking for these two patients. Cytomegalovirus was not detected (0/327).

Since a seasonal pattern of occurrence for EV and $\mathrm{HPeV}$ has been described cumulative detection rates were plotted from January through December (Fig. 1). For EV highest rates were observed in the summer months from June to September. No EV detections were seen in the years 1998, 1999, 2003 and 2006 (Table 1). The two HPeV cases were detected in October and November 2008, respectively. The HSV positive sample was detected in January 2008 and the VZV positive one in May 2007.

To further characterize the EV positive CSF samples the VP2 region was partially sequenced. All EV for which sequencing was successful $(n=10)$ belonged to $\mathrm{EV}$ species B. In detail, six samples were echovirus 30 , one was echovirus six, one echovirus 13 , one $\mathrm{CV}-\mathrm{B} 3$, and one was $\mathrm{CV}$-B6. Of note, in one patient in whom typing of the CSF sample was unsuccessful, EV-30 RNA could be identified in a corresponding stool sample. This sample was rated as EV-30 in all subsequent analyses. All EV-30 positive samples were randomly scattered throughout the entire study period. A total of three samples with EV concentrations of 3.3, 3.8, and 5.9 $\log 10$ RNA copies $/ \mathrm{ml}$, respectively, remained refractory to typing. Sequencing failed in CSF samples from two patients due to rather low RNA concentrations. In the sample with the highest viral RNA concentration no leftover impeded

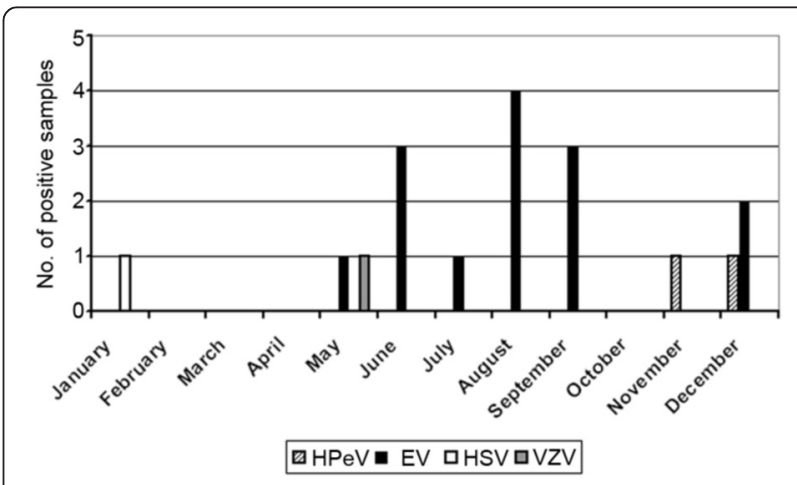

Fig. 1 Monthly distribution of positive samples. Shown is the cumulative number of positives samples in each month from January to December. Each viral pathogen detected (HPeV, EV, HSV, VZV) is indicated by a different bar 
Table 1 Proportion of samples positive for EV, HPeV, HSV, and VZV in CSF samples collected from 1998 to 2008

\begin{tabular}{llllll}
\hline & & \multicolumn{4}{l}{ Proportion (\%) of virus-positive samples } \\
\cline { 3 - 6 } Year & Number of patients & EV & HPeV & HSV & VZV \\
\hline 1998 & 11 & 0 & 0 & 0 & 0 \\
1999 & 12 & 0 & 0 & 0 & 0 \\
2000 & 27 & $3(11.1)$ & 0 & 0 & 0 \\
2001 & 27 & $1(3.7)$ & 0 & 0 & 0 \\
2002 & 31 & $1(3.2)$ & 0 & 0 & 0 \\
2003 & 23 & 0 & 0 & 0 & 0 \\
2004 & 24 & $3(12.5)$ & 0 & 0 & 0 \\
2005 & 34 & $1(2.9)$ & 0 & 0 & 0 \\
2006 & 42 & 0 & 0 & 0 & 0 \\
2007 & 47 & $2(4.2)$ & 0 & 0 & $1(2)$ \\
2008 & 49 & $3(6.1)$ & $2(4.1)$ & $1(2)$ & 0 \\
Total & 327 & $14(4.3)$ & $2(0.6)$ & $1(0.3)$ & $1(0.3)$ \\
\hline
\end{tabular}

typing. Sequencing of the VP1/VP3 region of the $\mathrm{HPeV}$ positive samples yielded $\mathrm{HPeV} 3$ in both cases. Both HPeV3 samples lacked a RGD motif in the VP1/2A junction region.

In a next step, virus RNA concentrations of EV and $\mathrm{HPeV}$ in CSF samples were analyzed. The mean virus concentration of the EV positive samples was $4.3 \log 10$ RNA copies/ml [95 \% confidence interval (CI) 3.9-4.7 $\log 10$ RNA copies/ml]. For further analysis two groups of EV-positive samples were compared. One group comprised samples with EV-30 $(n=7)$ and the second group all other non EV-30 EV samples $(n=4)$ in which typing was successful. Mean virus concentration in the first group was $4.4 \log 10$ RNA copies $/ \mathrm{ml}$, in the latter group $4.2 \log 10$ RNA copies/ml (Mann-Whitney Utest, $p>0.05$ ).

An HPeV RNA concentration of $5.3 \log 10$ RNA copies/ml was measured in a 5-week-old child and a concentration of $4.4 \log 10$ RNA copies/ml in a 1-week-old neonate, respectively. Next, viral loads between echovirus $30(n=7)$, all other typed non EV-30 EV positive samples $(n=4)$ and both $\mathrm{HPeV}$ positive samples were compared. Mean $\log 10$ viral RNA concentrations of EV-30, other typed non EV-30 EV, and HPeV (4.9 log10 RNA copies/ $\mathrm{ml}$ ) samples were not significantly different as assessed by one-way ANOVA (Fig. 2). Of note, mean interval between symptom onset and drawing of CSF was 0.4 days for EV positive samples and 4.5 days for $\mathrm{HPeV}$ positive samples ( $p=0.002$, two-tailed t-test). Of note, in the 5-week-old child with $\mathrm{HPeV}$ infection a second lumbar puncture was performed on day 7 after symptom onset upon clinical deterioration (periodic breathing, lack of spontaneous movement). The first lumbar puncture was performed on day 1 of symptoms (hypothermia, sucking weakness, and

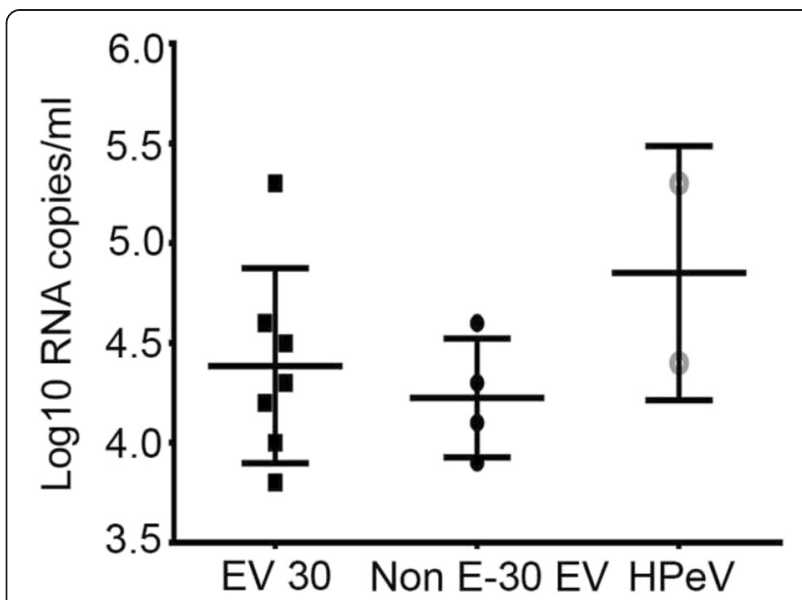

Fig. 2 Viral RNA concentrations. Log10 RNA concentration per ml CSF in samples positive for echovirus 30, non E-30 EV, and HPeV, respectively. Virus concentrations are given on a log scale on the y-axis. Each dot represents 1 specimen. Horizontal lines represent median values for each sample group. Outer horizontal lines represent the $95 \% \mathrm{Cl}$

reduced general condition) but CSF was used up for other testing. Mean virus concentrations of typed EV $(n=2)$ and $\mathrm{HPeV}(n=2)$ in children $<3$ months of age were not significantly different (4.5 log10 RNA copies/ml versus 4.9 $\log 10$ RNA copies/ml, $p<0.05$, two-tailed t-test).

Mean age of children with EV-30 infection was 5.1 years (range, $1-9), 1.6$ years (range, 3 months-6 years) for those with non EV-30 EV infection, and 3 weeks for those with $\mathrm{HPeV} 3$ infection. Aseptic meningitis was diagnosed in 12/14 (86 \%) patients with EV infection, but not in patients with $\mathrm{HPeV}$ infection (Table 2). The indication for lumbar puncture (i.e. aseptic meningitis or encephalitis or other neurological symptoms) could not unambiguously be retrieved for one child with EV infection, for another child with EV infection fever, headache, meningism without pleocytosis was recorded. No clinical signs of encephalitis were reported in those with EV infection, but in one child with $\mathrm{HPeV}$ infection. Clinically, both infants positive for $\mathrm{HPeV}$ displayed sepsis-like illness. In the 1-week-old neonate this included reduced general condition, sucking weakness, fever, and on day 2 tonic-clonic seizures. Cranial ultrasound showed hyperechogenicity, slurred gyrification, and hyperemia. The 5-week-old infant first presented with hypothermia, sucking weakness, reduced general condition, rash, and later developed respiratory distress. No fatal outcome was observed in the entire study cohort.

\section{Discussion}

In our cohort, we could show that HPeV3 is equally prevalent to EV in infants less than 3 months of age, which has not been observed in other studies so far. Previous studies showed a prevalence of $\mathrm{HPeV}$ in CSF 
Table 2 Proportion of clinical characteristics between patients with EV, echovirus 30, non EV-30 EV, and HPeV in CSF samples

\begin{tabular}{|c|c|c|c|c|}
\hline Characteristic & All EV $(n=14)$ & Echovirus $30(n=7)$ & Non EV-30 EV $(n=4)$ & $\operatorname{HPeV}(n=2)$ \\
\hline Fever/Hypothermia & $93 \%(13 / 14)$ & $86 \%(6 / 7)$ & $100 \%(4 / 4)$ & $100 \%(2 / 2)$ \\
\hline Meningism & $57 \%(8 / 14)$ & $71 \%(5 / 7)$ & $25 \%(1 / 4)$ & $0 \%(0 / 2)$ \\
\hline Headache & $57 \%(8 / 14)$ & $86 \%(6 / 7)$ & $25 \%(1 / 4)$ & na \\
\hline Photophobia & $21 \%(3 / 14)$ & $29 \%(2 / 7)$ & $0 \%(0 / 4)$ & na \\
\hline Sepsis-like illness & $0 \%(0 / 14)$ & $0 \%(0 / 7)$ & $0 \%(0 / 4)$ & $100 \%(2 / 2)$ \\
\hline Aseptic meningitis & $86 \%(12 / 14)$ & $86 \%(6 / 7)$ & $100 \%(4 / 4)$ & $0 \%(0 / 2)$ \\
\hline Encephalitis & $0 \%(0 / 14)$ & $0 \%(0 / 7)$ & $0 \%(0 / 4)$ & $50 \%(1 / 2)$ \\
\hline Respiratory tract symptoms & $50 \%(7 / 14)$ & $57 \%(4 / 7)$ & $25 \%(1 / 4)$ & $50 \%(1 / 2)$ \\
\hline Gastrointestinal symptoms & $64 \%(9 / 14)$ & $86 \%(6 / 7)$ & $50 \%(2 / 4)$ & $0 \%(0 / 2)$ \\
\hline Rash & $0 \%(0 / 14)$ & $0 \%(0 / 7)$ & $0 \%(0 / 4)$ & $50 \%(1 / 2)$ \\
\hline
\end{tabular}

na not applicable

samples ranging from 0 to $17 \%[11,12]$. However, studies differed by inclusion criteria, study design, and demographic characteristics. In our study we used an unbiased approach and included all CSF samples which were sent from the pediatric department for routine diagnostics. Walters et al. used a similar approach and reported a prevalence of $2.3 \%$ for $\mathrm{HPeV}$ among children $<3$ months of age, which is in close agreement to our results [2]. In contrast to other studies, we did not observe a 2-year seasonality for $\mathrm{HPeV}$ [3]. A different epidemiology in Germany cannot be ruled out but the samples size per year was rather small and prolonged storage may have influenced our results. This is supported by another study from our laboratory where other $\mathrm{HPeV}$ types were more frequently detected in respiratory tract and stool samples compared to $\mathrm{HPeV} 3$ [13].

Of note, both $\mathrm{HPeV}$ cases occurred in the fall of 2008, which contrasts with a report from another European country, where most infections were seen in the spring [11]. Recent data from the USA indicate that the peak season for $\mathrm{HPeV}$ detection was in the summer months [12]. The highly variable epidemiology of $\mathrm{HPeV}$ is supported by recent findings from South Korea, where the majority of cases were detected in spring in one study and in summer in another study $[14,15]$. However, both studies covered only a short period of time. Beyond the detection of $\mathrm{HPeV}$ in clinical samples using PCR seroepidemiological data is urgently needed to better appreciate the epidemiology and impact of $\mathrm{HPeV}$ in general and of $\mathrm{HPeV} 3$ in particular. As expected for EV we observed a peak of cases in the summer months.

Typing of HPeV samples showed exclusively HPeV3, which is consistent with other studies. In contrast, typing of EV samples showed a diversity of EV species B subtypes. A seroepidemiogical study from France demonstrated the predominance of EV species B from 2000 to 2004 and echovirus 30 was the most frequently detected EV, which is supported by our data [16].
Noteworthy, similar findings were reported from Germany [17]. We did not detect EV71 although this strain is reported to circulate in different European countries $[18,19]$. However, cases of hand, foot, and mouth disease are typically seen by general practitioners without laboratory testing. Therefore, information on EV71 is scarce supporting the notion that monitoring of EV diversity is an important issue not only for public health. It has been shown that genotyping can help to rapidly identify novel types which are associated with severe disease [5]. Interestingly, the detection of EV and $\mathrm{HPeV}$ has been demonstrated in sewage samples which might serve as a method to rapidly detect spread of EV strains [19]. In addition, recombination of EV is a common event and surveillance of sewage can also detect less pathogenic EV which otherwise remain unnoticed [19].

Importantly, $\mathrm{HPeV} 3$ infections tended towards higher RNA concentrations compared to infections with EV although the difference was not statistically significant. This finding was also seen when only children $<3$ months of age where included in the analysis. Of note, both $\mathrm{HPeV}$ samples were obtained significantly later after symptom onset compared to EV positive samples suggesting even higher HPeV3 RNA concentrations. It is speculative if a missing RGD motif in $\mathrm{HPeV} 3$ and a possible neurotropism account for higher RNA concentrations [20]. A variation in virulence as proposed in a study may also be responsible [21]. Besides intrinsic viral factors recent seroepidemiological studies have demonstrated that more than $30 \%$ of child-bearing aged women lack antibodies to HPeV3 [22]. Another study showed a low prevalence of HPeV3 specific neutralizing antibodies among the adult Dutch (10\%) and Finnish (13\%) population [23]. Of note, the authors speculated on difficulties in producing $\mathrm{HPeV} 3$ specific antibodies and the possibility of a test artefact. However, if the lack of passively transferred maternal HPEV3 antibodies renders neonates vulnerable to infection remains speculative. In contrast, antibodies to EV are 
widespread and might prevent EV replication to high titers. For EV, genotype-dependent RNA concentrations have been described recently. Volle and colleagues demonstrated higher virus concentration in echovirus 30 cases compared to those with echovirus six infection [24]. We could observe a trend to higher RNA concentrations in echovirus 30 cases but without statistical significance. Clearly, more patients are needed to confirm our findings.

Interestingly, Harvala et al. reported $\mathrm{HPeV}$ RNA concentrations $<500$ copies $/ \mathrm{ml}$ in CSF samples of young infants submitted for bacterial sepsis screening [25]. From the same study it should be noted that spill-over of $\mathrm{EV}$ and $\mathrm{HPeV}$, with low viral concentration in CSF, can occur from the blood and the mere detection of viral RNA in the CSF is not sufficient for a diagnosis of CSF infection, particularly if other indicative findings of CNS infection like pleocytosis are absent [25]. Unfortunately, we were not able to systematically retrieve white cell count in CSF samples as a marker for CNS infection due to the retrospective nature of the study. However, they could demonstrate higher RNA concentrations in blood samples suggesting that this type of sample should be preferred in sepsis evaluation. Choosing the best sample type for diagnosis was also addressed by another study which showed that stool samples were superior over CSF samples [26]. On the other hand, $\mathrm{HPeV}$ was shown to be also present in stool samples of healthy children indicating asymptomatic shedding after infection [27]. From these data it is obvious to alert clinicians that samples from multiples sites can considerably increase diagnostic yield. In our retrospective study we were not able to retrieve corresponding blood samples from our patients. However, we could show that typing was positive from stool samples in those patients in which typing from CSF samples failed for unknown reasons supporting the notion that multiple samples prove beneficial. From a technical point, the use of contemporary PCR assays is highly recommended. We could show that the detection rate for EV increase by a quarter upon use of recently published assay [10]. According to Dierssen et al. modifications of the primer and probe binding sites increased the detection of divergent echovirus 30 strains [10]. It should be noted that we might have missed cases of $\mathrm{HPeV}$ associated CNS infection due to low $\mathrm{HPeV}$ concentrations as virus concentrations below 500 copies/ml in CSF samples have been reported in infants with sepsis-like illness [25].

\section{Conclusions}

Here, we could show that HPeV3 infection of the central nervous system occurs predominantly in young infants. Unlike for EV only one $\mathrm{HPeV}$ type, i.e. $\mathrm{HPeV} 3$, was seen. Higher viral loads in HPeV3 infection might contribute to severity of illness and deserve further studies. Beyond the detection and assessment of viral RNA concentrations genotyping might prove beneficial not only for prognostic appreciation.

\section{Methods}

\section{Clinical specimens}

A total of 327 archived CSF samples from 327 patients had been collected from May 1998 until October 2008. No samples were available from May to September 1999. Specimens were originally submitted to the Institute of Virology, University Medical Centre Bonn for routine virological work-up due to suspected meningitis or encephalitis at the discretion of the treating physician. Further criteria to obtain CSF samples were other neurological symptoms, e.g. meningism, bulging fontanella, seizures, reduced vigilance, and late-onset sepsis. Bacterial testing was not systematically done and thus not reported here. All samples were anonymized and stored at $-20{ }^{\circ} \mathrm{C}$. Due to prolonged storage no attempts for virus isolation were made. Residual testing was approved by the ethics committee of Bonn University Medical Centre.

\section{Nucleic acid extraction}

For PCR samples were extracted in pools of five and tested. In case of a positive finding pools were resolved and samples were individually re-extracted and tested. Viral nucleic acids were purified by means of a viral RNA mini Kit (Qiagen, Hilden, Germany) with minor modifications. Due to little left-over of samples the input volume was set to $120 \mu \mathrm{l}$, and the elution volume to $100 \mu \mathrm{l}$.

\section{Real-time reverse-transcription-PCR (RT-PCR) and real-time PCR}

For EV RNA detection two assays were performed as described $[9,10]$. For $\mathrm{HPeV}$ detection the assays according to Baumgarte et al. and Nix et al. were used [28, 29]. All assays were of quantitive nature using RNA transcripts as described. In brief, amplicons of each real-time RT-PCR were cloned into pCR4 plasmid vector (Life Technologies, Karlsruhe, Germany) and in vitro RNA transcribed using the MEGAScript T7 in vitro transcription kit (Life Technologies) [28]. The detection of cytomegalovirus (CMV), herpes simplex virus 1/2 (HSV), and varizella zoster-virus (VZV) was done as described elsewhere [30-32].

\section{EV and $\mathrm{HPeV}$ typing}

Typing of EV and $\mathrm{HPeV}$ was conducted as described $[28,33]$. EV positive samples were sequenced in the VP2 gene [33]. For $\mathrm{HPeV}$ the complete VP1 region including the RGD motif and the VP3/VP1 junction region was sequenced according to Haravla et al. [3]. Sequencing was done on an ABI Prism 3130 using the Big-Dye Terminator kit (Applied Biosystems, 
Weiterstadt, Germany). Sequences were analyzed using Bio-Edit and by comparison with published sequences in NCBI GenBank.

\section{Statistics}

Statistical analysis was performed using the GraphPad Prism 6 software package (Graphpad, La Jolla, CA, USA).

\section{Competing interest}

The authors declare that they have no competing interest.

\section{Authors' contributions}

AMEH, CD, and MP participated in the study design. AM and AS cared for the patients and retrieved clinical information. SV, JFD carried out the real-time PCR and molecular typing. SV, AMEH, MP performed the data analysis and drafted the manuscript. All authors participated in writing and revising the manuscript. All authors have read and approved the final manuscript.

\section{Acknowledgments}

We are grateful to Ulrike Reber for excellent technical assistance.

\begin{abstract}
Author details
'Institute of Virology, University of Bonn Medical Centre, Sigmund-Freud Str. 25, 53105 Bonn, Germany. ${ }^{2}$ Department of Paediatrics, University of Bonn Medical Centre, Sigmund-Freud Str. 25, 53105 Bonn, Germany. ${ }^{3}$ Institute of Virology, Medical Center - University of Freiburg, Hermann-Herder Str. 11, 79104 Freiburg, Germany. ${ }^{4}$ Present address: Paediatric Oncology and Haematology, University of the Saarland, Homburg, Germany.
\end{abstract}

Received: 14 August 2015 Accepted: 16 November 2015 Published online: 25 November 2015

\section{References}

1. Wolthers KC, Benschop KS, Schinkel J, Molenkamp R, Bergevoet RM, Spijkerman IJ, et al. Human parechoviruses as an important viral cause of sepsislike illness and meningitis in young children. Clin Infect Dis. 2008;47:358-63.

2. Walters B, Penaranda S, Nix WA, Oberste MS, Todd KM, Katz BZ, et al. Detection of human parechovirus (HPeV)-3 in spinal fluid specimens from pediatric patients in the Chicago area. J Clin Virol. 2011;52:187-91.

3. Harvala H, Robertson I, McWilliam Leitch EC, Benschop K, Wolthers KC, Templeton $\mathrm{K}$, et al. Epidemiology and clinical associations of human parechovirus respiratory infections. J Clin Microbiol. 2008;46:3446-53.

4. Solomon T, Lewthwaite P, Perera D, Cardosa MJ, McMinn P, Ooi MH. Virology, epidemiology, pathogenesis, and control of enterovirus 71 . Lancet Infect Dis. 2010;10:778-90.

5. Greninger AL, Naccache SN, Messacar K, Clayton A, Yu G, Somasekar S, et al. A novel outbreak enterovirus D68 strain associated with acute flaccid myelitis cases in the USA (2012-14): a retrospective cohort study. Lancet Infect Dis. 2015;15(6):671-82.

6. Pfeiffer HC, Bragstad K, Skram MK, Dahl H, Knudsen PK, Chawla MS, et al. Two cases of acute severe flaccid myelitis associated with enterovirus D68 infection in children, Norway, autumn 2014. Euro Surveill. 2015;20(10):21062.

7. Harvala $\mathrm{H}$, Wolthers $\mathrm{KC}$, Simmonds P. Parechoviruses in children: understanding a new infection. Curr Opin Infect Dis. 2010;23:224-30.

8. Renaud C, Kuypers J, Ficken E, Cent A, Corey L, Englund JA. Introduction of a novel parechovirus RT-PCR clinical test in a regional medical center. J Clin Virol. 2011;51:50-3.

9. Verstrepen WA, Kuhn S, Kockx MM, Van De Vyvere ME, Mertens AH. Rapid detection of enterovirus RNA in cerebrospinal fluid specimens with a nove single-tube real-time reverse transcription-PCR assay. J Clin Microbiol. 2001; 39:4093-6.

10. Dierssen U, Rehren F, Henke-Gendo C, Harste G, Heim A. Rapid routine detection of enterovirus RNA in cerebrospinal fluid by a one-step real-time RT-PCR assay. J Clin Virol. 2008;42:58-64.

11. Harvala H, McLeish N, Kondracka J, McIntyre CL, McWilliam Leitch EC, Templeton $\mathrm{K}$, et al. Comparison of human parechovirus and enterovirus detection frequencies in cerebrospinal fluid samples collected over a 5-year period in edinburgh: HPeV type 3 identified as the most common picornavirus type. J Med Virol. 2011;83:889-96.
12. Sharp J, Harrison CJ, Puckett K, Selvaraju SB, Penaranda S, Nix WA, et al. Characteristics of young infants in whom human parechovirus, enterovirus or neither were detected in cerebrospinal fluid during sepsis evaluations. Pediatr Infect Dis J. 2013:32:213-6.

13. Eis-Hubinger AM, Eckerle I, Helmer A, Reber U, Dresbach T, Buderus S, et al. Two cases of sepsis-like illness in infants caused by human parechovirus traced back to elder siblings with mild gastroenteritis and respiratory symptoms. J Clin Microbiol. 2013;51:715-8.

14. Han TH, Chung JY, You SJ, Youn JL, Shim GH. Human parechovirus-3 infection in children, South Korea. J Clin Virol. 2013;58:194-9.

15. Seo JH, Yeom JS, Youn HS, Han TH, Chung JY. Prevalence of human parechovirus and enterovirus in cerebrospinal fluid samples in children in Jinju, Korea. Korean J Pediatr. 2015;58:102-7.

16. Antona D, Leveque N, Chomel JJ, Dubrou S, Levy-Bruhl D, Lina B. Surveillance of enteroviruses in France, 2000-2004. Eur J Clin Microbiol Infect Dis. 2007;26:403-12.

17. Roth B, Enders M, Arents A, Pfitzner A, Terletskaia-Ladwig E. Epidemiologic aspects and laboratory features of enterovirus infections in Western Germany, 2000-2005. J Med Virol. 2007;79:956-62.

18. Rabenau HF, Richter M, Doerr HW. Hand, foot and mouth disease: seroprevalence of Coxsackie A16 and Enterovirus 71 in Germany. Med Microbiol Immunol. 2010;199:45-51.

19. Harvala H, Calvert J, Van Nguyen D, Clasper L, Gadsby N, Molyneaux P, et al. Comparison of diagnostic clinical samples and environmental sampling for enterovirus and parechovirus surveillance in Scotland, 2010 to 2012. Euro Surveill. 2014;19(15)

20. Benschop K, Thomas X, Serpenti C, Molenkamp R, Wolthers K. High prevalence of human Parechovirus ( $\mathrm{HPeV}$ ) genotypes in the Amsterdam region and identification of specific $\mathrm{HPeV}$ variants by direct genotyping of stool samples. J Clin Microbiol. 2008;46:3965-70.

21. Verboon-Maciolek MA, Groenendaal F, Hahn CD, Hellmann J, van Loon AM, Boivin G, et al. Human parechovirus causes encephalitis with white matter injury in neonates. Ann Neurol. 2008;64:266-73.

22. Ito M, Yamashita T, Tsuzuki H, Takeda N, Sakae K. Isolation and identification of a novel human parechovirus. J Gen Virol. 2004;85:391-8.

23. Westerhuis B, Kolehmainen P, Benschop K, Nurminen N, Koen G, Koskiniemi $M$, et al. Human parechovirus seroprevalence in Finland and the Netherlands. J Clin Virol. 2013;58:211-5.

24. Volle R, Bailly JL, Mirand A, Pereira B, Marque-Juillet S, Chambon M, et al. Variations in cerebrospinal fluid viral loads among enterovirus genotypes in patients hospitalized with laboratory-confirmed meningitis due to enterovirus. J Infect Dis. 2014;210:576-84.

25. Harvala H, Griffiths M, Solomon T, Simmonds P. Distinct systemic and central nervous system disease patterns in enterovirus and parechovirus infected children. J Infect. 2014;69:69-74.

26. de Crom SC, Obihara CC, de Moor RA, Veldkamp EJ, van Furth AM, Rossen JW. Prospective comparison of the detection rates of human enterovirus and parechovirus RT-qPCR and viral culture in different pediatric specimens. J Clin Virol. 2013;58:449-54.

27. Kolehmainen P, Oikarinen S, Koskiniemi M, Simell O, Ilonen J, Knip M, et al. Human parechoviruses are frequently detected in stool of healthy Finnish children. J Clin Virol. 2012;54:156-61.

28. Baumgarte S, de Souza Luna LK, Grywna K, Panning M, Drexler JF, Karsten C, et al. Prevalence, types, and RNA concentrations of human parechoviruses, including a sixth parechovirus type, in stool samples from patients with acute enteritis. J Clin Microbiol. 2008:46:242-8.

29. Nix WA, Maher K, Johansson ES, Niklasson B, Lindberg AM, Pallansch MA, et al. Detection of all known parechoviruses by real-time PCR. J Clin Microbiol. 2008:46:2519-24.

30. Kessler HH, Muhlbauer G, Rinner B, Stelzl E, Berger A, Dorr HW, et al. Detection of Herpes simplex virus DNA by real-time PCR. J Clin Microbiol. 2000;38:2638-42.

31. Preiser W, Brink NS, Ayliffe U, Peggs KS, Mackinnon S, Tedder RS, et al. Development and clinical application of a fully controlled quantitative PCR assay for cell-free cytomegalovirus in human plasma. J Clin Virol. 2003;26:49-59.

32. Stocher M, Leb V, Bozic M, Kessler HH, Halwachs-Baumann G, Landt O, et al. Parallel detection of five human herpes virus DNAs by a set of real-time polymerase chain reactions in a single run. J Clin Virol. 2003;26:85-93.

33. Nasri D, Bouslama L, Omar S, Saoudin H, Bourlet T, Aouni M, et al. Typing of human enterovirus by partial sequencing of VP2. J Clin Microbiol. 2007:45:2370-9. 